\title{
Akt Inhibitors and COL11A1 in Epithelial Ovarian Carcinoma: A Short Note
}

\author{
Cheng-Yang Chou ${ }^{1}$ and Yi-Hui $\mathrm{Wu}^{2 *}$ \\ ${ }^{1}$ Department of Obstetrics and Gynecology, National Cheng Kung University Hospital, College of Medicine, National Cheng Kung University, Taiwan \\ 2Department of Medical Research, Chi Mei Medical Center, Liouying Campus, Tainan, Taiwan
}

*Corresponding author: Yi-Hui Wu, Ph.D, Department of Medical Research, Chi Mei Medical Center, Liouying Campus, Tainan, 736, Taiwan; E-mail: a80869@mail. chimei.org.tw

Received: October 14, 2019; Accepted: October 31, 2019; Published: October 31, 2019;

\section{Short Commentary}

Epithelial ovarian carcinoma (EOC) is the most lethal gynecologic malignancy. Currently, the treatment of patients with EOC usually includes surgery and chemotherapy [1]. The survival rate of patients with EOC remains low despite advances in surgical techniques and chemotherapy. One of the obstacles to the use of chemotherapy is drug resistance. To improve the survival rate, efforts must be made to overcome chemoresistance.

Akt, a key protein in the Akt/PI3K signaling pathway, is a serine/ threonine protein kinase that, once activated by phosphorylation, plays an important role in the process of malignant transformation [2]. The phosphorylated form of Akt (p-Akt) has been implicated in the induction of signals that affect cell apoptosis and the promotion of cell proliferation and invasiveness through mammalian target of rapamycin (mTOR) activation [3]. Investigations have shown that overexpressed p-Akt is associated with a poor prognosis of human cancer [4-6] that includes ovarian cancers [7-9]. Our recent report showed that patients with tumors overexpressing p-Akt had a poorer survival rate, and the p-Akt overexpression was associated with highgrade tumors and cancer death [10]. In addition, more patients with high p-Akt levels were allocated to the group of clinically defined chemoresistance, although this difference did not achieve statistical significance [10]. Therefore, p-Akt overexpression may be a common prognostic factor shared by multiple types of human cancers, and thus has the potential to be a therapeutic target of clinical significance.

Collagen type XI alpha 1 (COL11A1) belongs to the collagen family, which is the major component of the interstitial extracellular matrix. We previously found that COL11A1 plays an important role in EOC. Our results indicated that COL11A1 promotes tumor progression by up regulating the transforming growth factor- $\beta 1$ (TGF- $\beta 1$ )/matrix metalloproteinase-3 (MMP3) axis, through the involvement of the nuclear transcription factor Y subunit alpha (NFYA) binding site in the COL11A1 promoter, and predicts a poor clinical outcome in ovarian cancer patients [11]. We also found that COL11A1 promotes cancer cell sensitivity to anticancer drugs via activation of the Akt/c/EBP $\beta$ (CCAAT/enhancer-binding protein beta) pathway and attenuates phosphoinositide-dependent kinase 1 (PDK1) ubiquitination and degradation [12]. In addition, COL11A1 reduced chemotherapy-induced apoptosis through up regulating Twistrelated protein 1 (TWIST1)-mediated induced myeloid leukemia cell differentiation protein (Mcl-1) and growth arrest-specific 6 (GAS6) expression [13]. Our recent report indicated that SC66, an inhibitor of Akt and mTOR, inhibited COL11A1 expression and enhanced the sensitivity of cells to anticancer drugs through the dual suppression of c/EBP $\beta$ and NF-YA binding to the COL11A1 promoter [10].

A previous study [14] described that the Akt inhibitor MK-2206 enhances the efficacy of anticancer drugs in ovarian cancer cells. However, our results showed that COL11A1 mRNA expression and COL11A1 promoter activity were regulated by SC66, but not by MK-2206 [10]. We also found out that the expression of PDK1 was inhibited by SC66, but not by MK-2206 [10]. These results suggest that Akt inhibitors might exert their effect on Akt signaling through different mechanisms. Further investigation is required to explore the precise molecular mechanisms underlying Akt inhibitor-regulated Akt-related signaling.

\section{Conclusion}

The PI3K/Akt signaling pathway has become the focus of interest as a critical regulator of cancer cell survival, and a number of Akt pathway inhibitors with different efficacy and specificity have been identified. In our opinion, Akt inhibitors might exert their effect on Akt signaling through different mechanisms, and evaluation of PI3K/ Akt/mTOR pathway inhibitors is required to confirm the patterns of sensitivity observed in preclinical studies before they can be applied in the clinic.

Keywords: Akt inhibitor, Chemoresistance, Cisplatin, COL11A1, Epithelial Ovarian Carcinoma, Paclitaxel

\section{References}

1. Siegel R, Naishadham D, Jemal A (2012) Cancer statistics. CA Cancer J Clin 62: 10-29.

2. Nicholson $\mathrm{KM}$ and Anderson NG (2002) The protein kinase B/Akt signaling pathway in human malignancy. Cell Signal 14: 381-395.

3. Vivanco I and Sawyers CL (2002) The phosphatidylinositol 3-kinase AKT pathway in human cancer. Nat Rev Cancer 2: 489-501.

4. Perez-Tenorio G, Stal O (2002) Group SSBC. Activation of AKT/PKB in breast cancer predicts a worse outcome among endocrine treated patients. $\mathrm{Br} J$ Cancer 86: $540-545$. 
5. Massarelli E, Liu DD, Lee JJ, El-Naggar AK, Lo Muzio L, et al. (2005) Akt activation correlates with adverse outcome in tongue cancer. Cancer 104: 24302436.

6. Yoshioka A, Miyata H, Doki Y, Yasuda T, Yamasaki M, et al. (2008) The activation of Akt during preoperative chemotherapy for esophageal cancer correlates with poor prognosis. Oncol Rep 19:1099-1107.

7. Jia W, Chang B, Sun L, Zhu H, Pang L, et al. (2014) p-AKT over-expression may predict poor prognosis in ovarian cancer. Int J Clin Exp Pathol 7: 5940-5949.

8. Huang J, Zhang L, Greshock J, Colligon TA, Wang Y, et al. (2011) Frequent genetic abnormalities of the PI3K/AKT pathway in primary ovarian cancer predict patient outcome. Genes Chromosomes Cancer 50: 606-618.

9. Tanaka Y, Terai Y, Tanabe A, Sasaki H, Sekijima T, et al. (2011) Prognostic effect of epidermal growth factor receptor gene mutations and the aberrant phosphorylation of Akt and ERK in ovarian cancer. Cancer Biol Ther 11: 50-57.
10. Wu YH, Huang YF, Chen CC, Chou CY (2019) Akt inhibitor SC66 promotes cell sensitivity to cisplatin in chemo resistant ovarian cancer cells through inhibition of COL11A1 expression. Cell Death Dis 10: 322.

11. Wu YH, Chang TH, Huang YF, Huang HD, Chou CY (2014) COL11A1 promotes tumor progression and predicts poor clinical outcome in ovarian cancer. Oncogene 33: 3432-3440.

12. Wu YH, Chang TH, Huang YF, Chen CC, Chou CY (2015) COL11A1 confers chemoresistance on ovarian cancer cells through the activation of Akt $/ \mathrm{c} / \mathrm{EBP} \beta$ pathway and PDK1 stabilization. Oncotarget 6: 23748-23763.

13. Wu YH, Huang YF, Chang TH, Chou CY (2017) Activation of TWIST1 by COL11A1 promotes chemoresistance and inhibits apoptosis in ovarian cancer cells by modulating NF-kB-mediated IKK $\beta$ expression. Int J Cancer 141: 2305-2317.

14. Lin YH, Chen BY, Lai WT, Wu SF, Guh JH, et al. (2015) The Akt inhibitor MK2206 enhances the cytotoxicity of paclitaxel (Taxol) and cisplatin in ovarian cancer cells. Naunyn Schmiedebergs Arch Pharmacol 388: 19-31.

\section{Citation:}

